\title{
Non-alcoholic Fatty Liver and Vitamin D Levels in Extremely Obese Individuals: A Case-Control Study
}

\author{
Mohamed Sayed ${ }^{1}$, Ebthal Mahmoud Abozeid ${ }^{1}$ (D), Hoda M. Amin ${ }^{1}$, Naglaa M. Elsayed ${ }^{1}$, Yasser H. Nassar ${ }^{2}$, Hend A. Elsheimy ${ }^{1 *}$ (D) \\ ${ }^{1}$ Department of Internal Medicine, Faculty of Medicine, Cairo University, Giza, Egypt; ${ }^{2}$ Department of Medical Biochemistry and \\ Molecular Biology, Faculty of Medicine, Cairo University, Giza, Egypt
}

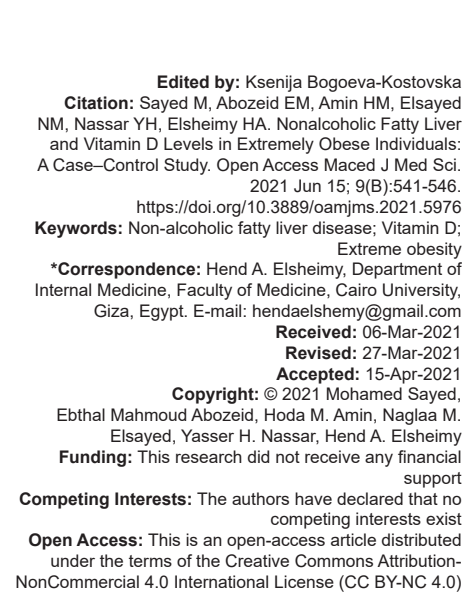

\section{Abstract}

BACKGROUND: Non-alcoholic fatty liver disease (NAFLD) is currently one of the most prevalent liver diseases. NAFLD is exemplified by the deposition of fat in the liver, in the absence of other etiologies. The spectrum of histological features in NAFLD ranges from macro-vesicular steatosis and nonalcoholic steatohepatitis, and it can eventually end in fibrosis, cirrhosis, or hepatocellular carcinoma. Vitamin D deficiency (VDD) is the most common micronutrient deficiency worldwide. Obese subjects are more prone to VDD, particularly those with liver disease. Non-classic functions of Vitamin D may be involved in the metabolic pathways beyond NAFLD development and progression.

AIM: The aim of the study was to evaluate the relationship between NAFLD and Vitamin D levels in extreme obese subjects, and the correlation between Vitamin D levels and NAFLD severity.

MATERIALS AND METHODS: The study included 80 Egyptian subjects of both sexes, divided into two groups: 50 patients with Stage III obesity (defined as body mass index $[\mathrm{BMI}] \geq 40 \mathrm{~kg} / \mathrm{m}^{2}$ ) and NAFLD, their age ranging from 20 to $<60$ years, and 30 age- and sex-matched healthy volunteers as control subjects. All patients were recruited from nutrition outpatient clinic at endocrinology unit, Cairo University Hospitals during the period from January 2019 to June 2019. Diagnosis of NAFLD was done by ultrasonography and laboratory evaluation included Vitamin D level. Nutritional evaluation included BMI, waist circumference, and weight.

RESULTS: Vitamin D was significantly lower in the NAFLD group versus healthy controls: About $34 \%$, deficient $32 \%$ insufficient, and $34 \%$ sufficient in NAFLD group versus $23.3 \%$ insufficient, and $76.7 \%$ sufficient only in control, $p<0.001$. The severity of NAFLD, as graded by ultrasonography, was positively correlated with BMI and inversely correlated with Vit $D$ levels, $p=0.001$ and 0.024 , respectively. Multivariate linear regression proved that both BM and Vit D were independent predictors for NAFLD progression, BMI in a positive manner and Vit D in a negative manner. A cutoff Vit D level of 27.75 had $64 \%$ sensitivity and $90 \%$ specificity in NAFLD detection, area under the curve was 0.821

CONCLUSION: Vitamin D is significantly lower in the NAFLD group versus healthy controls in this cohort. VDD and BMI were associated with increased NAFLD severity. VDD was found to be an independent predictor of NAFLD progression. Vitamin D supplementation may be added to lifestyle modifications to prevent NAFLD occurrence in obese subjects.

\section{Introduction}

Non-alcoholic fatty liver disease (NAFLD) is currently one of the most prevalent liver diseases [1], and it is exemplified by the deposition of fat in the liver, in the absence of other etiologies, for example, alcohol abuse, and viral hepatitis [2]. The spectrum of histological features in NAFLD ranges from macrovesicular steatosis and non-alcoholic steatohepatitis (NASH), and it can eventually end in fibrosis, cirrhosis, or hepatocellular carcinoma [3]. Various metabolic pathways are implicated in the development of NAFLD, and its progression from steatosis up to cirrhosis and hepatocellular carcinoma, this is known as the two-hit hypothesis [4].

NAFLD affects nearly $30 \%$ of the population in general, in patients with diabetes or obesity the prevalence increases up to $90 \%$. Insulin resistance, obesity, type II diabetes, hypertension, and dyslipidemia are strongly associated with NAFLD, and NFLD is now marked as a hepatic manifestation of the metabolic syndrome [5], [6].

Vitamin D deficiency (VDD) can be the result of defects in the absorption or hydroxylation of Vitamin $D$ due to impaired liver or kidney function, decreased dietary intake, or lack of sunlight exposure. It is the most common micronutrient deficiency worldwide, with nearly 1 billion of people affected [7]. Obese subjects are more prone to VDD, particularly those with liver disease [8], possibly due to the impaired synthesis of $25(\mathrm{OH})$ vitamin from the liver due to steatosis.

As our knowledge regarding Vitamin D functions expand, it became accepted that Vitamin $D$ has an important role in cell differentiation, immune regulation, and inflammatory response. Some of these functions, 
non-classic functions of Vitamin $D$, are involved in the metabolic pathways beyond NAFLD development and progression [9].

In fact, Vitamin D intracellular signals are mediated by Vitamin $\mathrm{D}$ receptor (VDR), which are expressed in hepatocytes, VDD can aggravate NAFLD through an inflammatory-mediated pathway [10]. The expression of VDR in NAFLD hepatocytes is inversely correlated with the disease severity [11]. VDD may also share in the pathogenesis of NAFLD as it interferes with lipid metabolism by the liver [12]. Hence, the effectiveness of Vitamin $D$ replacement in patients with, or at high risk of NAFLD has become the topic of growing research [9].

Our work aimed to study the relationship between NAFLD and Vitamin D status in extremely obese patients, and the correlation between Vit $D$ levels and NAFLD severity.

\section{Materials and Methods}

\section{Patient population}

Our study is a case-control study that included 80 subjects who were divided into two groups. Group 1 included fifty Egyptian male and female patients with Stage III obesity, and NAFLD. Their age ranged from 20 to $<60$ years. Group 2 included 30 age- and sex-matched healthy volunteers as control subjects. All patients were recruited from nutrition outpatient clinic at endocrinology unit, Cairo University Hospitals during the period from January 2019 to June 2019. The study protocol was approved by the Internal Medicine Department Ethical Committee. An informed consent was obtained from all participants before their enrollment.

The inclusion criteria were, male and female patients, age above 20 and $<60$ years, Stage III obesity and NAFLD. The following categories were excluded from participating in the study; women who are pregnant or lactating, patients with clinical manifestations of and or who are diagnosed with malabsorption syndrome, patients suffering from acute or chronic infections, diabetic patients, adrenal insufficiency, or hypothyroidism, subjects receiving Vitamin D treatment, consuming alcohol or hepatotoxic drugs, and patients with history of chronic liver disease.

\section{Data collection}

All participating individuals were subjected to:

- Detailed history and physical examination, including measurement of waist circumference, which was measured while the patient was standing, feet together, arms by the sides, abdomen relaxed, and measured by a nonextensible tap. Height was measured in $\left(\mathrm{m}^{2}\right)$ and weight in $(\mathrm{kg})$ to calculate the body mass index (BMI), where Stage III obesity was defined as $\mathrm{BMI} \geq 40 \mathrm{Kg} / \mathrm{m}^{2}$ [13]

Routine biochemical tests including for assessment of liver enzymes, lipid profile, and blood glucose.

- Totalserum25-hydroxyvitaminD(VitaminD):We used Human 25-Dihydroxy Vitamin D human ELISA kit to measure Vitamin $D$ in the sera our study subjects. The kit is based on double antibody sandwich technique, it was provided by Sunredbio, Shanghai, China [14]. Vitamin D levels were classified into sufficient $(\geq 30$ to $<100 \mathrm{ng} / \mathrm{ml}$ ), insufficient (20 to $29.9 \mathrm{ng} / \mathrm{ml}$ ), and deficient $(<20 \mathrm{ng} / \mathrm{ml}$ ), this classification was based on Endocrine Society guidelines [15]

Diagnosis of NAFLD by ultrasonography was performed using a 2-5 $\mathrm{MHz}$ convex transducer. We classified our patients as having different grades of steatosis (0-3) according to the liver echogenicity. Grade I: Liver echogenicity is mildly increased, and Grade 2: The liver echogenicity obscures the branches of the portal vein and Grade 3, where the echogenicity obscures the diaphragmatic outline [16]. We diagnosed NAFLD when the score was $>$ or $=2$ on the Hamaguchi et al. 2007 ultrasonographic scoring system [17].

\section{Statistical methods}

Data were coded and entered using the Statistical Package for the Social Sciences version 25 (IBM Corp., Armonk, NY, USA). We summarized quantitative variables using mean and standard deviation, for categorial variables we used frequencies and relative frequencies. Comparison between different groups was done using unpaired t-test. We used Chi-square $\left(\chi^{2}\right)$ to compare categorical data, when the frequency was expected to be $<5$ we used Exact test instead. The correlation between quantitative variables was performed using Pearson correlation coefficient. Linear regression analysis was done to predict independent predictors of NAFLD degree in obese cases. Logistic regression was done to detect independent predictors of NAFLD. $p<0.05$ was considered as statistically significant.

\section{Results}

Our study population was divided into two groups. Group I (cases) included 50 extremely obese 
$(B M I \geq 40)$ patients with NAFLD, $20(40 \%)$ of them were males, and $30(60 \%)$ females with their age ranging from $\geq 20$ to $<60$ years. Group II (control) included 30 healthy volunteers, $14(47.7 \%)$ of the control group were males and $16(53.3 \%)$ females, with matching age. Weight, $\mathrm{BMI}$, and waist circumferences were all higher in cases in comparison to controls, the difference was highly significant $(p<0.001)$.

Platelet count and high-density lipoprotein (HDL) levels were significantly lower in the cases in comparison with the control subjects with $p<0.001$. On contrary, total bilirubin, international normalized ratio (INR), serum aspartate transaminase (AST), Alanine transaminase (ALT), fasting blood sugar (FBS), total $\mathrm{CHOL}$, low-density lipoprotein (LDL), and triglycerides levels were significantly higher in cases in comparison to controls, also with a highly significant statistical difference $(p<0.001)$, these data are presented in Table 1.

Table 1: Comparison of the different laboratory variables between the two studied groups

\begin{tabular}{lllllll}
\hline Variables & Cases & & & Controls & p-value (t-test) \\
\cline { 2 - 3 } & Mean & SD & & Mean & SD & \\
\hline HB & 12.01 & 1.61 & & 11.82 & 0.94 & 0.559 \\
TLC & 7.17 & 1.69 & & 7.75 & 1.87 & 0.158 \\
PLT & 243.32 & 86.63 & & 315.33 & 81.98 & $<0.001$ \\
AST & 51.46 & 32.54 & & 24.00 & 9.35 & $<0.001$ \\
ALT & 53.56 & 31.36 & & 24.00 & 9.73 & $<0.001$ \\
Bil (T) & 1.00 & 0.22 & & 0.84 & 0.21 & 0.002 \\
Bil (D) & 0.31 & 0.12 & & 0.30 & 0.19 & 0.728 \\
Albumin & 3.98 & 0.25 & & 3.94 & 0.77 & 0.744 \\
INR & 1.07 & 0.09 & & 1.00 & 0.00 & $<0.001$ \\
FBS & 94.72 & 11.64 & & 83.43 & 6.84 & $<0.001$ \\
Urea & 17.70 & 5.40 & & 16.2 & 5.2 & 0.612 \\
Creatinine & 0.80 & 0.24 & & 0.85 & 0.19 & 0.783 \\
ALP & 75.6 & 18.7 & & 71.97 & 20.91 & 0.279 \\
GGT & 27.2 & 12.5 & & 26.37 & 10.25 & 0.821 \\
Chol & 244.32 & 47.73 & & 137.10 & 27.19 & $<0.001$ \\
LDL & 144.34 & 27.62 & 90.13 & 12.07 & $<0.001$ \\
HDL & 43.50 & 8.42 & & 69.03 & 17.19 & $<0.001$ \\
TGs & 221.12 & 97.82 & & 65.77 & 22.53 & $<0.001$ \\
Vit D & 26.34 & 11.28 & 44.12 & 15.70 & $<0.001$ \\
\hline HB: Hemoglobin, & TLC: Total leukocyte count, PLT: Platelet, AST: Aspartate transaminase, ALT: Alanine
\end{tabular}
HDL: High-density lipoprotein, TGs: Triglycerides, Vit D: Vitamin D.

Levels of Vitamin D were significantly lower in cases (26.34 \pm 11.28 ) compared with controls (44.12 \pm 15.7). Further analysis of Vitamin D levels in the studied groups revealed that 17 cases $(34 \%)$ were deficient, 16 (32\%) were insufficient, and 17 (34\%) had sufficient Vit D levels; on the other hand, the control Group had only seven (23.3\%) subjects with insufficiency, $23(76.7 \%)$ with sufficiency, and none of the participants were deficient in Vit D. These differences were statistically significant $(p<0.001)$, Tables 1 and Figure 1 demonstrate these data.

In our obese cases, we did not detect any significant correlation between Vitamin D levels and clinical variables including age, weight, waist circumference, and BMI. Furthermore, no significant correlation was found with other laboratory variables including hemoglobin, total leukocytic count, platelets, AST, ALT, billirubin, albumin, INR, fasting blood glucose (FBG), urea, creatinine, total cholesterol, LDL cholesterol, HDL cholesterol, and triglycerides.

According to the abdominal ultrasound, all obese cases were suffering from NAFLD. We found

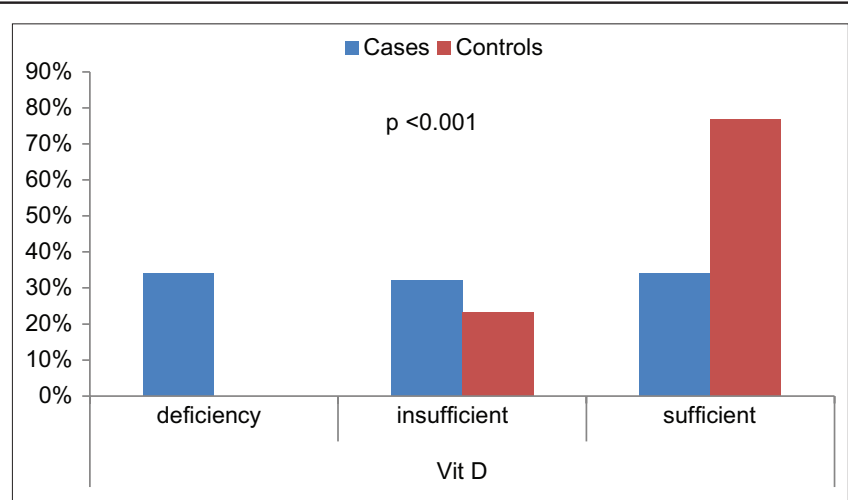

Figure 1: Comparison between cases and control as regarding Vitamin $D$ level

that $40 \%$ of them had Grade 2, 32\% had Grade 3, and $28 \%$ had Grade 1 fatty changes. None of our control subjects suffered from NAFLD.

The degree of NAFLD by ultrasonography was positively correlated with weight and BMI, both with a highly significant statistical difference. There was no significant correlation with age, height or waist circumference, these data are demonstrated in Table 2.

Table 2: Correlation between vitamin NAFLD degree and clinical variables in cases

\begin{tabular}{lll}
\hline NAFLD (U/S) & $\mathrm{r}$ & $\mathrm{p}$-value \\
\hline Age & 0.258 & 0.071 \\
Waist circumference & 0.246 & 0.085 \\
WT & 0.428 & 0.002 \\
Height & 0.212 & 0.139 \\
BMI & 0.460 & 0.001 \\
\hline BMI: Body mass index, NAFLD: Non-alcoholic fatty liver disease, WT: Weight.
\end{tabular}

NAFLD was negatively correlated with Vit D levels and positively correlated with platelet count in the cases (Group I); the correlation was statistically significant with a p-value of 0.024 and 0.035 , respectively. On contrary, there was no significant correlation with other laboratory parameters Table 3.

Multivariable regression analysis was done within the NAFLD group to test for potential independent predictors of disease progression as marked by ultrasound grades. The ultrasound grade was used as a continuous variable. Potential factors

Table 3: Correlation between NAFLD ultrasonographic grades and different laboratory variables in patients with extreme obesity

\begin{tabular}{lll}
\hline NAFLD (U/S) & $r$ & $p$-value \\
\hline Vit D & -0.318 & 0.024 \\
HB & 0.064 & 0.657 \\
TLC & 0.056 & 0.697 \\
PLT & 0.298 & 0.035 \\
AST & -0.008 & 0.956 \\
ALT & 0.016 & 0.914 \\
Bil(T) & 0.058 & 0.688 \\
Bil(D) & -0.028 & 0.849 \\
Albumin & 0.045 & 0.756 \\
INR & 0.195 & 0.175 \\
FBS & -0.145 & 0.316 \\
Urea & -0.113 & 0.434 \\
Creatinine & -0.010 & 0.943 \\
Chol & 0.211 & 0.142 \\
LDL & 0.064 & 0.661 \\
HDL & -0.257 & 0.071 \\
TGs & 0.222 & 0.121 \\
\hline HB: Hemoglobin, TLC: Total leukocyte count, PLT: Platelet, AST: Aspartate transaminase, ALT: Alanine \\
transaminase, INR: International normalized ratio, FBS: Fasting blood sugar, LDL: Low-density lipoprotein, \\
HDL: High-density lipoprotein, TGs: Triglycerides, Vit D: Vitamin D.
\end{tabular}


that were entered into the model include age, gender, waist circumference, lipid profile, liver function tests, blood picture parameters FBG, and Vitamin D level. Insignificant variables by univariable analysis were excluded from the study. BMI and Vitamin D prove to be independent predictors of NAFLD severity. BMI in a positive manner and Vitamin $D$ in a negative manner is shown in Table 4.

Table 4: Multivariable linear regression to detect predictors of NAFLD degree in patients with extreme obesity

\begin{tabular}{llllll}
\hline Independent variables & Coefficient & Std. Error & $\mathrm{t}$ & $\mathrm{p}$ & rpartial \\
\hline BMI & 0.09402 & 0.02493 & 3.771 & 0.0005 & 0.4985 \\
PLT & 0.001891 & 0.001053 & 1.796 & 0.0796 & 0.2641 \\
TGs & 0.001277 & 0.0009063 & 1.409 & 0.1661 & 0.2100 \\
Vit_D & -0.02130 & 0.007842 & -2.717 & 0.0095 & -0.3828 \\
Waist circumference & -0.002610 & 0.005335 & -0.489 & 0.6272 & -0.07439 \\
\hline BMI: Body mass index, PLT: Platelet, TGs: Triglycerides, Vit D: Vitamin D. & &
\end{tabular}

We performed receiver operating characteristic (ROC) curve on the studied groups, both NAFLD and non-NAFLD. ROC curve showed that at a cutoff 27.75 of Vitamin $D$, the sensitivity to predict the presence of NAFLD is $64 \%$, the specificity to exclude the presence of disease is $90 \%$, and the area under the curve (AUC) was 0.821 .

\section{Discussion}

In our study, most of the patients are females constituting $60 \%$, a previous research showed similar results, where the percent of females seeking advice in weight control clinics were as high as $80 \%$, thus gender is not a confounding factor in our work [18].

In our study, Vitamin D levels were significantly lower in obese cases with NAFLD compared with control subjects. In obese NAFLD group, 32\% were insufficient, $34 \%$ deficient, and $34 \%$ sufficient versus $23.3 \%$ insufficient and $76.7 \%$ sufficient in control, demonstrating a highly significant statistical difference between the two studied groups $p<0.001$.

There was no significant difference between the two studied groups regarding the synthetic liver functions. Hence, lower Vit D levels in the cases cannot be attributed to diminished hepatic synthesis of Vit D.

These results agree with a previous metaanalysis including 17 case-control and cross-sectional studies, the result of which revealed that Vitamin D levels were lower in patients with NAFLD. VDD was associated with 1.26 fold increase in the risk of NAFLD, compared with non-deficient subjects suggesting an important role of Vitamin D in NAFLD development [19].

Our results are also in agreement with Targher et al. [20], Targher et al. [21] who similarly detected lower levels of Vitamin D in NAFLD patients compared with normal individuals.
On contrary to our results, no association between Vitamin D levels and NAFLD in the results of Patel et al. [22], Paula et al. [23]. Variations in nutrition state or study population may explain the opposing results.

In the present study, AST and ALT levels were significantly higher in the cases in comparison to the control group (mean 51.46 and 53.56 vs. 24 and 24, p $<0.001$ ), levels. Our results in this concern agree with variable previous results, including [24] where ALT was significantly higher in NAFLD patients.

In our current work, NAFLD ultrasonographic grades were inversely correlated with Vitamin $D$, and positively correlated with BMI, the correlation was statistically significant $(r=-0.318$ and $p=0.024)$ and $(r=0.460$ and $p=0.001)$, respectively.

Interestingly, there was no significant correlation between vit $D$ level and BMI, $r=0.035, p=0.811$. Hence, Vit $D$ may have a direct effect on NAFLD that is independent from BMI.

Multivariate linear regression performed to detect independent predictors of NAFLD progression demonstrated that $\mathrm{BMI}$ and Vitamin $\mathrm{D}$ prove to be independent predictors of NAFLD severity. BMI in a positive manner and Vitamin $D$ in a negative manner. Therefore, VDD is associated with more severe NAFLD. It may have a causative role in its progression that is not related to obesity.

Similarly, Vitamin D level was inversely related to the degree of liver steatosis and fibrosis, with Vitamin D levels of $25.1 \pm 0.29 \mathrm{ng} / \mathrm{mL}$ for normal ultrasonogaphy, $24.7 \pm 0.42 \mathrm{ng} / \mathrm{mL}$ for mild steatosis, $23.7 \pm 0.37 \mathrm{ng} / \mathrm{mL}$ for moderate steatosis, and $23.6 \pm 0.60 \mathrm{ng} / \mathrm{mL}$ for sever steatosis, $(p<0.001)$ [25].

The previous studies have also demonstrated that the expression of VDR is inversely correlated with NASH severity, in terms of inflammatory stages [11].

The exact pathogenesis of NAFLD development and deterioration in VDD is not fully understood. Theories include the lack Vitamin D anti-inflammatory and antifibrotic effect on hepatic stellate cells. As Vitamin D decreases peripheral and hepatic insulin resistance induced by free fatty acids, VDD could be associated with increased accumulation of lipids in the liver and hence NAFLD development [26].

In our study, ROC curve used to predict NAFLD using Vitamin D level in obese patients; at a cutoff point $\leq 27.75$ of Vitamin $D$, its sensitivity to predict the presence of NAFLD is $64 \%$, and its specificity to exclude the presence of disease is 90, AUC 0.821 and $p<0.000$, these results are shown in Figure 2. A higher cutoff point $\leq 40$ had $77.17 \%$ sensitivity and $89.36 \%$ specificity of predicting NAFLD, AUC 0.749 and $p<0.000$ in a previous study [27]. Another recent study revealed a much lower cutoff point, where serum 


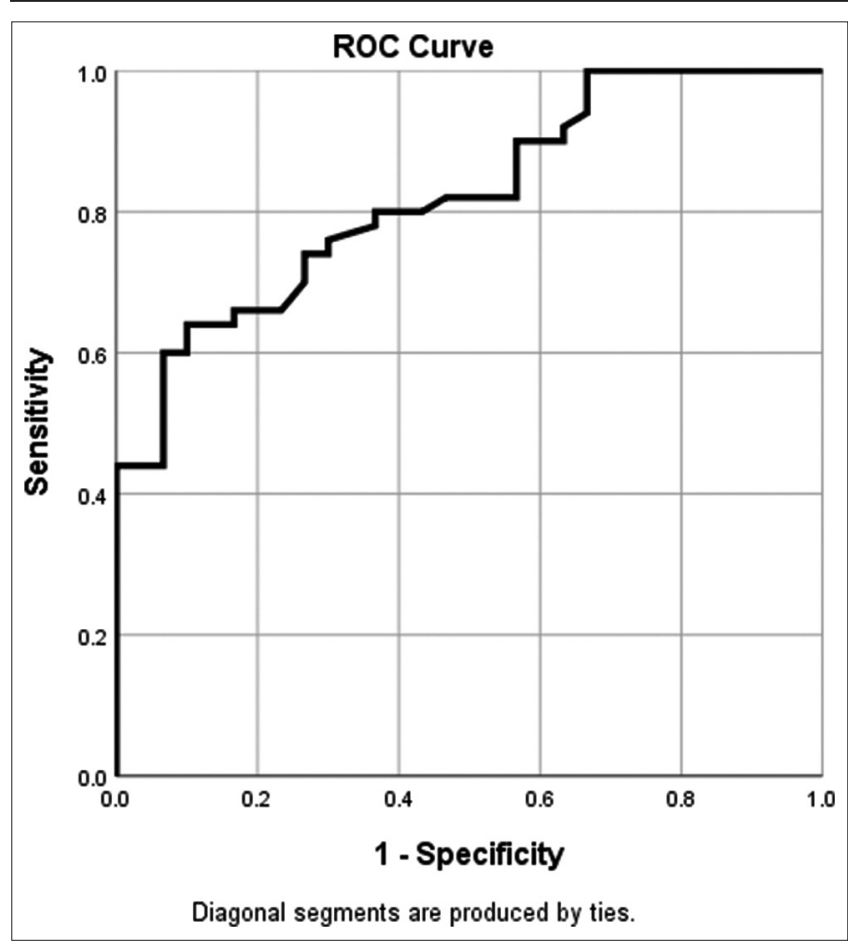

Figure 2: Receiver operating characteristic curve used to predict nonalcoholic fatty liver disease (NAFLD) using Vitamin D level, at a cutoff 27.75 of Vitamin D, $64 \%$ sensitivity to predict and $90 \%$ specificity to exclude NAFLD, area under the curve $=0.821$

$25(\mathrm{OH}) \mathrm{D}$ level of $<11.2 \mathrm{ng} / \mathrm{ml}$ increases the risk of NAFLD with $45 \%$ sensitivity and $97.5 \%$ specificity [26].

\section{Conclusion}

Extremely obese NAFLD patients included in our study had significantly lower Vitamin $D$ levels compared with healthy volunteers. Furthermore, Vit $D$ level was inversely correlated with NAFLD severity. A cut off 27.75 of Vitamin D, had $64 \%$ sensitivity and $90 \%$ specificity in NAFLD prediction.

\section{Limitations}

The lack of liver biopsy and the dependence on ultrasonography on diagnosis and grading of NAFLD. Even though biopsy is the gold standard, we choose ultrasonography as a safe, non-invasive, and reliable method in NAFLD assessment.

\section{Recommendations}

The authors recommend correction of Vitamin D level in all NAFLD patients. This may aid in preventing the disease progression. Further prospective and interventional studies are needed to delineate the cause-effect relationship between VDD and NAFLD. Further studies are also needed to determine the effect of Vitamin D treatment on NAFLD progression.

\section{Acknowledgments}

We appreciate all the participants in this work.

\section{Data Availability}

The datasets generated and/or analyzed during the current study are available from the corresponding author on reasonable request.

\section{References}

1. Chalasani N, Younossi ZM, Lavine JE, Diehl AM, Brunt EM, Cusi K. The diagnosis and management of non-alcoholic fatty liver disease: Practice guideline by the American association for the study of liver diseases, American college of gastroenterology, and the American gastroenterological association. Hepatology. 2012;55(6):20. https://doi.org/10.1002/hep.25762

PMid:22488764

2. Armstrong MJ, Adams LA, Canbay A, Syn WK. Extrahepatic complications of nonalcoholic fatty liver disease. Hepatology. 2014;59(3):1174-97. https://doi.org/10.1002/hep.26717 PMid:24002776

3. Vernon G, Baranova A, Younossi ZM. Systematic Review: The epidemiology and natural history of non-alcoholic fatty liver disease and non-alcoholic steatohepatitis in adults. Aliment Pharmacol Ther. 2011;34(3):274-85. https://doi. org/10.1111/j.1365-2036.2011.04724.x

PMid:21623852

4. Benedict $M$, Zhang $X$. Non-alcoholic fatty liver disease: An expanded review. World J Hepatol. 2017;9(16):715-32. https:// doi.org/10.4254/wjh.v9.i16.715

PMid:28652891

5. McCullough AJ. Epidemiology of the metabolic syndrome in the USA. J Dig Dis. 2011;12(5):333-40.

PMid:21091931

6. Nakahara $\mathrm{T}$, Hyogo $\mathrm{H}$, Yoneda $\mathrm{M}$, Sumida $\mathrm{Y}$, Eguchi $\mathrm{Y}$, Fujii $\mathrm{H}$, et al. Type 2 diabetes mellitus is associated with the fibrosis severity in patients with nonalcoholic fatty liver disease in a large retrospective cohort of Japanese patients. J Gastroenterol. 2014;49(11):1477-84. https://doi.org/10.1007/ s00535-013-0911-1

PMid:24277052

7. Wacker M, Holiack MF. Vitamin D-effects on skeletal and extraskeletal health and the need for supplementation. Nutrients. 2013;5(1):111-48. https://doi.org/10.3390/nu5010111 PMid:23306192 
8. Entrenas AO, Tubio DL, Navarroet FL, Carvajal FM, Osinaga JA. Relationship between Vitamin $D$ deficiency and the components of metabolic syndrome in patients with morbid obesity, before and 1 year after laparoscopic roux-en-Y gastric bypass or sleeve gastrectomy. Obesity Surg. 2017;27(5):1222-8. https:// doi.org/10.1007/s11695-016-2445-0

PMid:27864670

9. Kwok RM, Torres DM, Harrison SA. Vitamin D and nonalcoholic fatty liver disease (NAFLD): Is it more than just an association? Hepatology. 2013;58(3):1166-74. https://doi.org/10.1002/ hep. 26390 PMid:23504808

10. Han S, Li T, Ellis E, Strom S, Chiang JY. A novel bile acidactivated Vitamin $D$ receptor signaling in human hepatocytes. Mol Endocrinol. 2010;24(6):1151-64. https://doi.org/10.1210/ me.2009-0482

PMid:20371703

11. Barchetta I, Carotti S, Labbadia G, Gentilucci UV, Muda AO, Angelico $\mathrm{F}$, et al. Liver Vitamin $\mathrm{D}$ receptor, CYP2R1, and CYP27A1 expression: Relationship with liver histology and Vitamin D3 levels in patients with nonalcoholic steatohepatitis or hepatitis C virus. Hepatology. 2012;56(6):2180-7. https://doi. org/10.1002/hep.25930

PMid:22753133

12. Norman AW. From Vitamin D to hormone D: Fundamentals of the Vitamin $D$ endocrine system essential for good health. Am J Clin Nutr. 2008;88(2):491S-9. https://doi.org/10.1093/ ajcn/88.2.491s

PMid:18689389

13. World Health Organization. World Health Statistics 2011 Geneva: World Health Organization; 2014. [Last accessed on 2020 June 10]. Available from: http://www.who.int/healthinfo/ statistics/en/index.html.

14. Lequin RM. Enzyme immunoassay (EIA)/enzyme-linked immunosorbent assay (ELISA). Clin Chem. 2005;51(12):2415-8. https://doi.org/10.1373/clinchem.2005.051532 PMid:16179424

15. Holick MF, Binkley NC, Bischoff-Ferrari HA, Gordon CM, Hanley DA, Heaney RP, et al. Evaluation, treatment, and prevention of Vitamin D deficiency: An endocrine society clinical practice guideline. J Clin Endocrinol Metab. 2011;96(7):1911-30. https://doi.org/10.1210/jc.2011-0385 PMid:21646368

16. Saadeh S, Younossi ZM, Remer EM, Gramlich T, Ong JP, Hurley $\mathrm{M}$, et al. The utility of radiological imaging in nonalcoholic fatty liver disease. Gastroenterology. 2002;123(3):745-50. https://doi.org/10.1053/gast.2002.35354 PMid:12198701

17. Hamaguchi M, Kojima T, Itoh $\mathrm{Y}$, Harano $\mathrm{Y}$, Fujii K, Nakajima T, et al. The severity of ultrasonographic findings in nonalcoholic fatty liver disease reflects the metabolic syndrome and visceral fat accumulation. Am J Gastroenterol. 2007;102(12):2708-15. https://doi.org/10.1111/j.1572-0241.2007.01526.x

\section{PMid: 17894848}

18. Benoit SC, Hunter TD, Francis DM, De La Cruz-Munoz N. Use of bariatric outcomes longitudinal database (BOLD) to study variability in patient success after bariatric surgery. Obes Surg. 2014;24(6):936-43. https://doi.org/10.1007/s11695-014-1197-y PMid:24570089

19. Eliades M, Spyrou E, Agrawal N, Lazo M, Brancati FL, Potter JJ, et al. Meta-analysis: Vitamin $D$ and non-alcoholic fatty liver disease. Alimentary Pharmacol Therapeut. 2013;38(3):246-54. https://doi.org/10.1111/apt.12377 PMid:23786213

20. Targher G, Bertolini L, Scala L, Cigolini M, Zenari L, Falezza G, et al. Associations between serum 25-hydroxyvitamin D3 concentrations and liver histology in patients with non-alcoholic fattyliverdisease. NutrMetab CardiovascDis. 2007;17(7):517-24 . https://doi.org/10.1016/j.numecd.2006.04.002 PMid: 16928437

21. Dasarathy J, Periyalwar P, Allampati S, Bhinder V, Hawkins C Brandt $\mathrm{P}$, et al. Hypovitaminosis $\mathrm{D}$ is associated with increased whole body fat mass and greater severity of non-alcoholic fatty liver disease. Liver Int. 2014;34(6):e118-27. https://doi. org/10.1111/liv. 12312

PMid:24118743

22. Patel YA, Henao R, Moylan CA, Guy CD, Piercy DL, Diehl AM, et al. Vitamin $D$ is not associated with severity in NAFLD: Results of a paired clinical and gene expression profile analysis. Am J Gastroenterol. 2016;111(11):1591-8. https://doi.org/10.1038/ ajg.2016.406

PMid:27644736

23. Paula FV, Leandra R, de Paula Francisco A, Martinelli AD. Low Vitamin $D$ level is not associated with severity of non-alcoholic fatty liver disease in morbidly obese patients. J Hepatol. 2017;66:S157. https://doi.org/10.1016/s0168-8278(17)30588-3

24. Kasapoglu B, Turkay C, Yalcin KS, Carlioglu A, Sozen M, Koktener A. Low Vitamin D levels are associated with increased risk for fatty liver disease among non-obese adults. Clin Med. 2013;13(6):576-9. https://doi.org/10.7861/ clinmedicine.13-6-576

PMid:24298105

25. Kim HS, Rotundo L, Kothari N, Kim SH, Pyrsopoulos N Vitamin $D$ is associated with severity and mortality of nonalcoholic fatty liver disease: A US population-based study. J Clin Transl Hepatol. 2017;5(3):185-92. https://doi.org/10.14218/ jcth.2017.00025

PMid:28936398

26. Gad Al, Elmedames MR, Abdelhai AR, Marei AM. The association between Vitamin $D$ status and non-alcoholic fatty liver disease in adults: A hospital-based study. Egypt Liver J. 2020;10:25. https://doi.org/10.1186/s43066-020-00033-z

27. Mohamed AA, Mahmoud S, Ahmed R, Ahmed R, Fadl H, Atallah MA, et al. Vitamin D is it a key in Egyptian NAFLD pathogenesis? J Gastroenterol Hepatol. 2015;4:1605-9. https:// doi.org/10.17554/j.issn.2224-3992.2015.04.522 